\title{
miR-494 inhibits cervical cancer cell proliferation through upregulation of SOCS6 expression
}

\author{
LEI CHENG $^{1,2}$, BEIHUA KONG ${ }^{1}$, YING ZHAO ${ }^{2}$ and JIE JIANG ${ }^{1}$ \\ ${ }^{1}$ Department of Obstetrics and Gynecology, Qi Lu Hospital of Shandong University Jinan, \\ Jinan, Shandong 250012; ${ }^{2}$ Department of Obstetrics and Gynecology, Qi Lu Hospital of \\ Shandong University Qingdao, Qingdao, Shandong 266000, P.R. China
}

Received December 14, 2015; Accepted July 5, 2017

DOI: $10.3892 / \mathrm{ol} .2017 .7651$

\begin{abstract}
It is unclear how microRNA (miR)-494 inhibits the proliferation of cervical cancer cells by altering the expression of SOCS6. Therefore, the present study aimed to investigate the molecular mechanism underlying miR-494 regulation of suppressor of cytokine signaling 6 (SOCS6) in human cervical cancer samples and the human cervical cancer HeLa cell line. The expression of miR-494 was determined using reverse transcription-quantitative polymerase chain reaction. In addition, TargetScan was used to predict miR-494 target genes and the luciferase reporter assay was used to determine whether SOCS6 was a direct target of miR-494. The results of the present study demonstrated that compared with the cervical intraepithelial neoplasia and normal cervical tissues, the miR-494 expression level in cervical cancer samples was significantly decreased $(\mathrm{P}<0.01)$. In addition, compared with normal cervical tissue, miR-494 expression level was significantly decreased in cervical intraepithelial lesions $(\mathrm{P}<0.05)$. Furthermore, the expression of miR-494 was associated with patients with or without lymph node metastasis, clinical stage and depth of stromal invasion $(\mathrm{P}<0.01)$; however, miR-494 expression was not identified to be associated with age, tumor size and menopausal status $(\mathrm{P}>0.05)$. Transfection of a miR-494 mimic significantly increased the expression level of miR-494 in HeLa cells $(\mathrm{P}<0.01)$, and anti-miR-494 transfection decreased the expression of miR-494 $(\mathrm{P}<0.01)$. An MTT proliferation assay and Boyden chamber invasion ability assay revealed that miR-494 mimic transfection significantly inhibited the proliferation, and invasion ability of HeLa cells $(\mathrm{P}<0.01)$, whereas anti-miR-494 transfection significantly increased the proliferation and invasion ability $(\mathrm{P}<0.05)$. SOCS6 was predicted, using bioinformatics, to be the target
\end{abstract}

Correspondence to: Dr Jie Jiang, Department of Obstetrics and Gynecology, Qi Lu Hospital of Shandong University Jinan, 107 Wenhua Xi Road, Jinan, Shandong 250012, P.R. China E-mail: jiangjie20150904@sina.com

Key words: microRNA-494, suppressor of cytokine signaling 6, cervical cancer, proliferation, invasion gene of miR-494 and this was validated using a luciferase reporter assay. Western blot analysis revealed that transfection of miR-494 significantly increased the expression of SOCS6 in HeLa cells, and transfection of anti-miR-494 significantly decreased the expression of SOCS6. Therefore, the results of the present study demonstrated that miR-494 expression in cervical cancer was significantly decreased. Exhibiting a decreased expression level of miR-494 may result in enhanced proliferative and invasive abilities of HeLa cell, thus contributing to the occurrence, and development of cervical cancer.

\section{Introduction}

Cervical cancer is the second most common type of female malignancy, subsequent to breast cancer. There were an estimated 527,600 new cervical cancer cases and 265,700 deaths worldwide in 2012 (1). Although cervical cytology screening has enabled early detection and early treatment of cervical cancer, the incidence and mortality rates continue to increase each year $(2,3)$. The majority of patients with cervical cancer are treated with standard radiation therapy and chemotherapy, but therapeutic response varies. Thus, studies are required to determine the pathogenesis of cervical cancer in order to identify a more effective treatment for cervical cancer.

MicroRNAs (miRNA/miR) are endogenous non-coding RNAs containing between 19 and 21 nucleotides. miRNA may regulate proteins at the post-transcriptional level, resulting in the degradation or inhibition of proteins. miRNAs are involved in the regulation of a number of biological functions, including cell cycle, proliferation, differentiation and apoptosis. Previous studies have revealed abnormal expression of a number of miRNAs in cervical cancer, including miR-214, miR-21, miR-143 and miR-145 (4-7). In cervical cancer, miR-214 expression is decreased, which has been reported to inhibit cancer progression through targeting mitochondrial transcription factor A (8). miR-21 overexpression may inhibit the expression of phosphatase and tensin homolog (PTEN), and promote cancer cell proliferation and migration (9). In addition, miR-143 expression was identified to be significantly decreased in cervical cancer, and associated with tumor size, positive lymph node metastasis and HPV16 infection (10). A previous study demonstrated that the expression level of miR-145 was significantly decreased in human cervical cancer 
tissues when compared with corresponding adjacent normal tissues and associated with tumor progression, and poor prognosis (11). Furthermore, miR-494 serves as a tumor suppressor miRNA and abnormal expression of miR-494 has been identified in a number of tumor tissue types $(8,9)$. However, whether the expression level of miR-494 is altered in cervical cancer remains unknown. In addition, there are few detailed reports focusing on the role of miR-494 in cervical cancer.

The present study analyzed the expression of miR-494 in cervical tissue using reverse transcription-quantitative polymerase chain reaction (RT-qPCR), its association with clinicopathological characteristics of cervical cancer, and its effects on HeLa cells through transfection with anti-miR-494 and miR-494 mimics. The present study aimed to identify the effect of miR-494 on HeLa cell viability and invasion. Additionally, the present study aimed at exploring the association between miR-494 and cervical cancer development, metastasis and invasion, in order to identify novel therapeutic targets.

\section{Materials and methods}

Patients and tissue samples. Cervical cancer tissues were selected from 40 patients with cervical cancer who were admitted to the Qi Lu Hospital of Shandong University (Qingdao, China) between December 2011 and December 2014. No patients previously received preoperative chemotherapy or radiotherapy. Patients age range was between 25-72 years; mean age 52.23 \pm 20.13 years. All cervical cancer tissue samples were validated by independent pathologists. Clinical and pathological classification and staging were performed according to the International Federation of Gynecology and Obstetrics criteria (10). The present study additionally included 40 cervical intraepithelial lesions and 40 matched normal cervical tissues as the control group. All specimens were stored in liquid nitrogen within $30 \mathrm{~min}$ of resection. All protocols performed in the present study were approved by the Ethics Committee of the Qi Lu Hospital of Shandong University. Written informed consent was obtained from all patients.

Reagents and equipment. The following equipment and reagents were utilized in the present study: $\mathrm{CO}_{2}$ incubator (Thermo Fisher Scientific, Inc., Waltham, MA, USA); pipette (Eppendorf, Hamburg, Germany); weighing balance; inverted microscope; Milli-Q plus ultra-pure water system (EMD Millipore, Billerica, MA, USA); cervical cancer cell line HeLa (Institute of Basic Medical Chinese Academy of Medical Sciences, Beijing, China); miR-494 mimic and anti-miR-494 (Invitrogen; Thermo Fisher Scientific, Inc.), RPMI 1640 medium (Gibco; Thermo Fisher Scientific, Inc.); fetal bovine serum (Hyclone; GE Healthcare Life Sciences, Logan, UT, USA), TRIzol (Invitrogen; Thermo Fisher Scientific, Inc.); ready-to-use PCR kit (BBI solutions, Cardiff, UK); primers (Sangon Biotech Co., Ltd., Shanghai, China). Cells were cultured in RPMI-1640 medium containing $10 \%$ fetal bovine serum at $37^{\circ} \mathrm{C}$ in a $5 \% \mathrm{CO}_{2}$ incubator.

$R T-q P C R$. Total RNA was extracted from tissues using TRIzol reagent (Invitrogen; Thermo Fisher Scientific, Inc.) according to the manufacturer's protocol. cDNAs were synthesized with the Prime-Script RT reagent kit (Takara Bio, Inc., Otsu, Japan). The qPCR reaction conditions were as follows: $95^{\circ} \mathrm{C}$ for $5 \mathrm{~min}$, $95^{\circ} \mathrm{C}$ for $10 \mathrm{sec}$ and $60^{\circ} \mathrm{C}$ for $20 \mathrm{sec}$, for 40 cycles. The primer sequences (11) were as follows: miR-494 forward, 5'-TGACCT GAAACATACACGGGA-3' and miR-494 reverse, 5'-TAT CGTTGTACTCCACTCCTTGAC-3'. GADPH was used as the internal reference gene and the primers used were as follows: Forward, 5'-CGGAGTCAACGGATTTGGTCGTAT-3'; and reverse, 5'-AGCCTTCTCCATGGTGGTGAAGAC-3'. Data was subjected to quantitative analysis by comparing the $\mathrm{Cq}$ values and using the $2^{-\Delta \Delta \mathrm{Cq}}$ method (12).

Cell transfection methods. HeLa cells were seeded in a 24 -well plate at a density of $4 \times 10^{4}$ cells/well. Prior to transfection, all transfection reagents were at room temperature and medium was placed into incubator for $30 \mathrm{~min}$. Following $48 \mathrm{~h}$ transfection at $37^{\circ} \mathrm{C}$, cells were washed with PBS containing $1 \%$ bovine serum albumin (Sigma Aldrich; Merck KGaA, Darmstadt, Germany) twice and resuspended with $100 \mu \mathrm{l}$ Nucleofector ${ }^{\circledR}$ electroporation (Invitrogen; Thermo Fisher Scientific, Inc.). Subsequently, the corresponding RNA was added (300 pmol/sample). The cell suspension was placed into an electroporation cuvette, tapped to ensure that there were no air bubbles at the bottom of the electrical rotor, and the electroporation program was selected and run according to the manufacturer's protocol. Cells were immediately taken out of the electrical rotor, transferred into 12-well plates and cultured in the incubator.

MTT viability assay. The transfected cells $\left(0.5 \times 10^{4}\right.$ cells/well $)$ were seeded in 96-well culture plates and incubated with $20 \mu \mathrm{l}$ MTT at $37^{\circ} \mathrm{C}$ for $4 \mathrm{~h}$. Subsequently, the supernatant was aspirated and 15 ul dimethyl sulfoxide solution was added. After 10 min of low agitation, the optical density was determined at a wavelength of $490 \mathrm{~nm}$.

Invasion assay. Following $48 \mathrm{~h}$ transfection, $0.25 \%$ trypsin (containing EDTA) was used to digest cells. Cells were stained with $0.16 \%$ trypan blue dye solution for $3 \mathrm{~min}$ at room temperature. Cells $\left(5 \times 10^{5}\right)$ were suspended in serum-free RPMI 1640 medium and the lower layer of the Transwell chamber was plated with RPMI 1640 medium, supplemented with $10 \%$ fetal bovine serum. Cells were plated in the upper chamber and incubated at $37^{\circ} \mathrm{C}$ in an atmosphere containing $5 \% \mathrm{CO}_{2}$ for $24 \mathrm{~h}$. The membrane was coated with Matrigel (BD Bioscience, San Jose, CA, USA). Subsequently, the number of invaded cells were counted in 16 fields of view. Images were captured at a wavelength of $490 \mathrm{~nm}$ on a Leica DC 300F camera (Leica Microsystems GmbH, Wetzlar, Germany).

Bioinformatics prediction of miR-494 target genes. TargetScan (http://www.targetscan.org/vert_71/) target gene prediction software selected suppressor of cytokine signaling 6 (SOCS6) as a target gene of miR-494. TargetScan target gene prediction software identified the 3726-3733 site at the 3' end of the untranslated region (3'UTR) of SOCS6 mRNA as a possible site of action of miR-494.

Dual-luciferase reporter assay. The 3'UTR of SOCS6, containing putative miR-99a-binding sites was amplified 


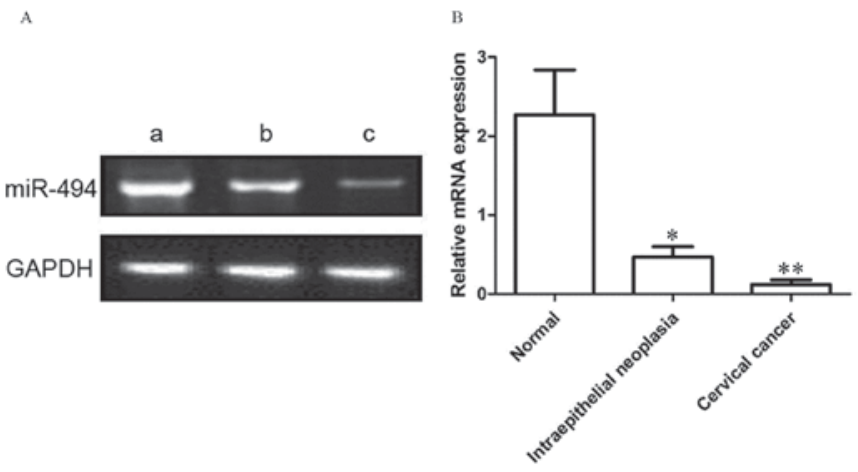

Figure 1. miR-494 expression levels in normal, intraepithelial neoplasia and cervical cancer tissues, determined using the reverse transcription-quantitative polymerase chain reaction. GADPH was used as the control. (A) Representative blot and (B) quantification of miR-494 expression. (Aa), normal cervical tissue resected non-cancerous tissues; $(\mathrm{Ab})$, cervical intraepithelial neoplasia tissues; (Ac), cervical cancer tissues. miR, microRNA. ${ }^{*} \mathrm{P}<0.05 ;{ }^{* *} \mathrm{P}<0.01$ vs. normal cervical tissue.

and cloned into pMIR (Ambion; Thermo Fisher Scientific, Inc.). In vitro synthesized DNA fragment containing the position and DNA fragment containing the mutant site, cloned into dual luciferase start after a child carrier pMIR. PMIR carrier (Ambion, Austin, TX, USA) and miR494 were co-transfected into HeLa cells using Dharmafect Duo transfection reagent (Thermo Fisher Scientific, Inc.); the cells were collected $48 \mathrm{~h}$ post-transfection. The PMIR carrier containing wild-type and mutated full-length 3'UTR from human SOCS6 (RefSeq NM_004232.3), was purchased from Labomics (GeneCopoeia, Inc., Rockville, MD, USA). The sense siRNA sequence was GCUGCGAUAUCAACGGUGAtt; the antisense siRNA sequence was UCACCGUUGAUAUCGCAGCtg. Luciferase activity was measured using the Dual-Luciferase Reporter Assay system (Promega Corporation) according to the manufacturer's recommendations. The luciferase activity was detected on a GLOMAX20/20 luminometer (Promega Corporation) and normalized to the Renilla luciferase activity.

Western blot analysis. Total protein was extracted from cells using the protein extraction reagent (Novagen; Merck KGaA) accordingly to the manufacturer's protocol and total protein concentration was determined using a BCA Protein Assay kit (Thermo Fisher Scientific, Inc.). A total of $50 \mu \mathrm{g}$ extracted protein per lane was separated using SDS-PAGE (8-10\% gel) and $5 \%$ stacking gel isolated, and transferred to nitrocellulose membranes. Subsequently, membranes were blocked at room temperature with Tris-Buffered Saline-Tween-20 (TBST) containing $5 \%$ bovine serum albumin for $1 \mathrm{~h}$. Membranes were incubated with anti- $\beta$-actin (1:5,000; cat. no. A5441; Sigma-Aldrich; Merck KGaA) or SOCS6 antibody (1:1,000; cat. no. ab53181; Abcam, Cambridge, UK) overnight at $4{ }^{\circ} \mathrm{C}$. The following day, membranes were washed with $0.1 \%$ TBST 3 times (5 min each), the horseradish peroxidase-labeled-anti-rat serum (1:1,000; Dako; Agilent Technologies, Inc., Santa Clara, CA, USA) secondary antibody was added and incubated at room temperature for $1 \mathrm{~h}$. Subsequently, membranes were washed with $0.1 \%$ TBST and Supersignal West Femto HRP sensitive chemiluminescent substrate (Pierce; Thermo Fisher Scientific, Inc.) was added to visualize the bands. $\beta$-actin was
Table I. miR494 expression level and clinicopathological features of cervical cancer.

\begin{tabular}{|c|c|c|c|c|}
\hline Parameter & $\mathrm{n}$ & $\operatorname{miR}-494$ & t-value & P-value \\
\hline Age, years & & & 0.356 & 0.736 \\
\hline$<40$ & 21 & $2.28 \pm 0.79$ & & \\
\hline$\geq 40$ & 19 & $2.19 \pm 0.71$ & & \\
\hline Tumor diameter, $\mathrm{cm}$ & & & 0.177 & 0.866 \\
\hline$<2.5$ & 20 & $2.17 \pm 0.72$ & & \\
\hline$\geq 2.5$ & 20 & $2.22 \pm 0.67$ & & \\
\hline Menopause & & & 0.106 & \\
\hline Yes & 18 & $2.25 \pm 0.52$ & & 0.919 \\
\hline No & 22 & $2.41 \pm 0.50$ & & \\
\hline Clinical stage & & & 5.556 & 0.001 \\
\hline I-II & 19 & $2.93 \pm 0.44$ & & \\
\hline III-IV & 21 & $1.11 \pm 0.61$ & & \\
\hline Interstitial infiltration & & & 5.444 & 0.002 \\
\hline$<2 / 5$ & 24 & $2.65 \pm 0.48$ & & \\
\hline$\geq 2 / 5$ & 16 & $1.29 \pm 0.72$ & & \\
\hline Lymph node metastasis & & & 5.261 & 0.002 \\
\hline No & 22 & $2.79 \pm 0.57$ & & \\
\hline Yes & 18 & $1.44 \pm 0.62$ & & \\
\hline
\end{tabular}

t-test was used. miR, microRNA.

used as the internal control. All experiments are representative of a minimum of three independent repeats.

Statistical analysis. Data are presented as the mean \pm standard deviation. SPSS software (version 16.0; SPSS, Inc., Chicago, IL, USA) was used for statistical data analysis. $\mathrm{P}<0.05$ was considered to indicate a statistically significant difference. Differences between miR-494 expression and clinicopathological features of cervical cancer were assessed using Student's t-test (unpaired). The differences in expression between three groups were examined by one-way analysis of variance followed by a Dunnett's post hoc test.

\section{Results}

miR-494 expression is decreased in cervical cancer. As presented in Fig. 1, the relative expression level of miR-494 was $0.12 \pm 0.06,0.47 \pm 0.13$ and $2.27 \pm 0.57$ in cervical cancer, cervical intraepithelial neoplasia and normal cervical tissues, respectively. Compared with the cervical intraepithelial neoplasia, the miR-494 expression level in cervical cancer samples was significantly decreased $(\mathrm{P}<0.01)$. In addition, compared with normal cervical tissue, miR-494 expression level was significantly decreased in cervical intraepithelial lesions $(\mathrm{P}<0.05)$.

miR-494 expression is associated with clinicopathological features of cervical cancer. As presented in Table I, the expression level of miR-494 in patients with cervical cancer was associated with clinical stage, depth of stromal invasion 


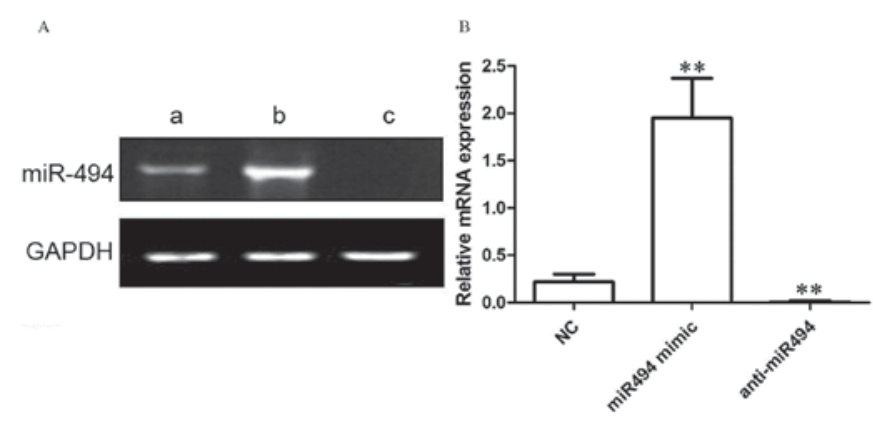

Figure 2. miR-494 expression level in a control group relative to that following transfection with miR-494 and anti-miR-494, determined using reverse transcription-quantitative polymerase chain reaction. GADPH was used as the control. (A) Representative blot and (B) quantification of miR-494 expression. (Aa), control group; (Ab), transfection miR-494 mimic group; (Ac), transfection of anti-miR-494. miR, microRNA; NC, negative control. ${ }^{*} \mathrm{P}<0.05 ;{ }^{* *} \mathrm{P}<0.01$ vs. NC.

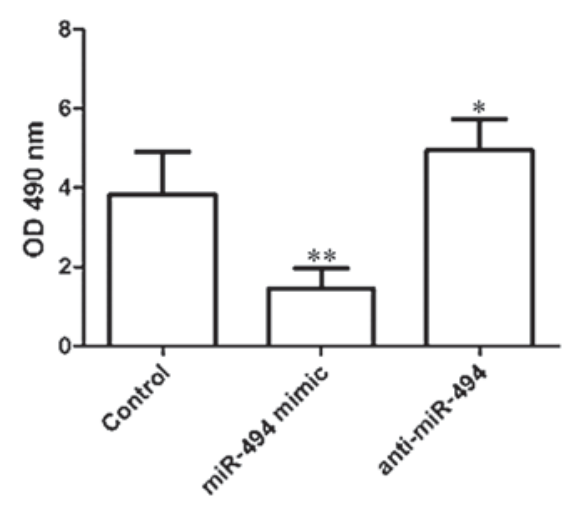

Figure 3. Viability of HeLa cells, determined using an MTT assay. Cells were transfected with either the control, the miR-494 mimic or the anti-miR-494. miR-494, microRNA; OD, optical density. ${ }^{*} \mathrm{P}<0.05 ;{ }^{* *} \mathrm{P}<0.01$ vs. the control group

and lymph node metastasis $(\mathrm{P}<0.01)$, but not with age, tumor diameter, and menopause $(\mathrm{P}>0.05)$.

miR-494 mimic transfection increases the expression level of miR-494. As presented in Fig. 2, in NC-transfected HeLa cells, the relative expression level of miR-494 was $0.22 \pm 0.08$. Following transfection with the miR-494 mimic, the relative expression level of miR-494 in HeLa cells was $1.95 \pm 0.42$. Following transfection with the anti-miR-494 in HeLa cells, the relative expression level of miR-494 was $0.01 \pm 0.01$. Compared with control group, miR-494 mimic transfection significantly increased the expression level of miR-494 $(\mathrm{P}<0.01)$, and anti-miR494 transfection significantly inhibited the expression level of miR-494 $(\mathrm{P}<0.01)$.

Transfection with the miR-494 mimic inhibits the viability of HeLa cells. As presented in Fig. 3, the viability of HeLa cells was determined to be $3.83 \pm 1.08,1.44 \pm 0.52$ and $4.94 \pm 0.79$ in the control, miR-494 mimic, and anti-miR494 groups, respectively. Compared with the control group, transfection with the miR-494 mimic significantly inhibited the viability of HeLa cells $(\mathrm{P}<0.01)$, and transfection with the anti-miR-494 significantly increased the viability of HeLa cells $(\mathrm{P}<0.05)$.

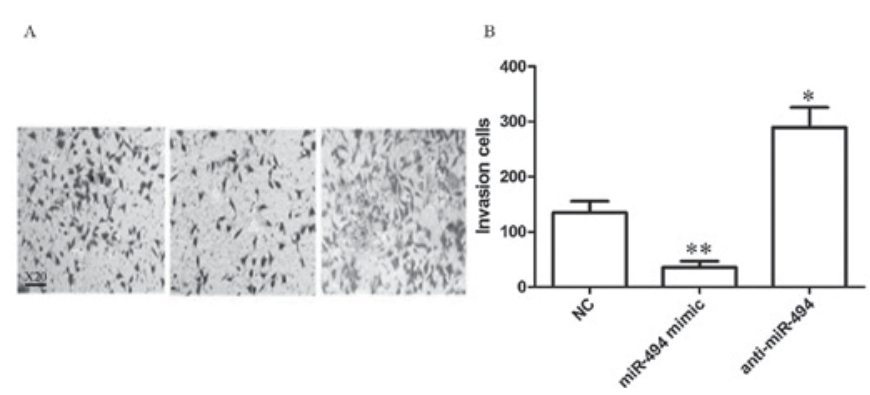

Figure 4. miR-494 affects the invasive ability of HeLa cells. (A) Representative images and (B) quantification of invasive cells. a, control group; b, transfection miR-494 mimic group; c, transfection of anti-miR-494 group. Magnification, $\mathrm{x} 20$. miR, microRNA; $\mathrm{NC}$, negative control. ${ }^{*} \mathrm{P}<0.05$; ${ }^{* * *} \mathrm{P}<0.01$ vs. the control group

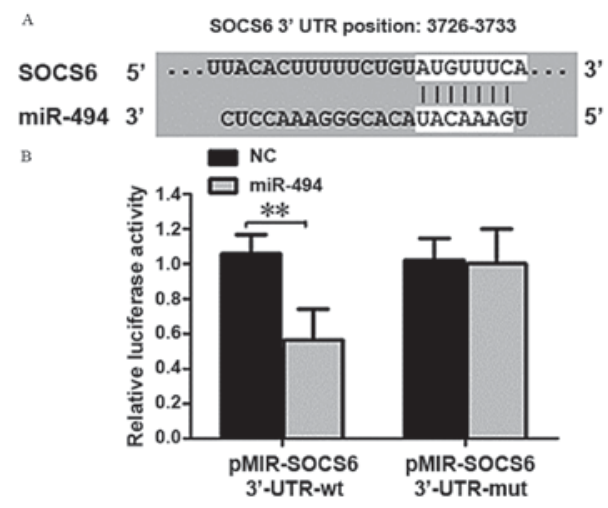

Figure 5. miR-494 target genes predicted using TargetScans software. (A) The forecasting SOCS6 3'-UTR target sites of miR-494. (B) Relative luciferase activity between wild type/mutant pMIR-SOCS6 3'-UTR carrier and miR-494. ${ }^{* *} \mathrm{P}<0.01$ vs. the NC group. miR, microRNA; SOCS6, suppressor of cytokine signaling 6; UTR, untranslated region; wt, wild-type; mut, mutant.
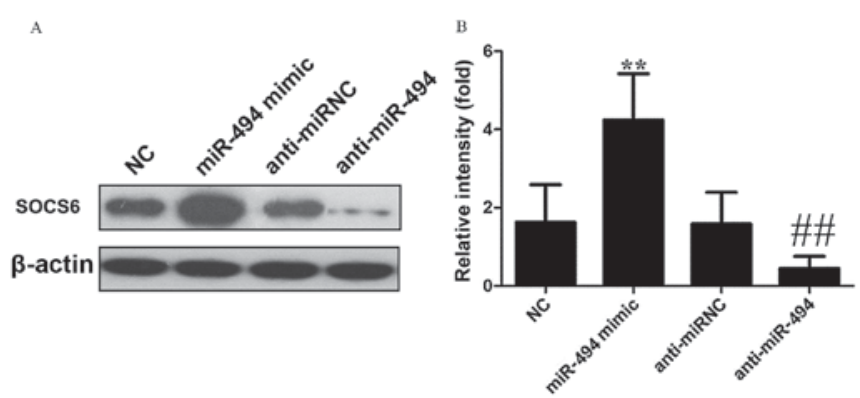

Figure 6. Western blot analysis and relative intensity following transfection of HeLa cells with miR-494 minic and anti-miR-494. (A) Western blot analysis. $\beta$-actin was used as a loading control. (B) Relative intensity. ${ }^{* *} \mathrm{P}<0.01$ vs. NC; ${ }^{\# \#} \mathrm{P}<0.01$ vs. anti-miR-NC. miR, microRNA; NC, negative control; SOCS6, suppressor of cytokine signaling 6 .

Transfection with miR-494 mimic inhibits the invasive ability of HeLa cells. As presented in Fig. 4, the number of invasive HeLa cells was $134.84 \pm 20.75,35.75 \pm 11.04$ and $289.45 \pm 35.94$ in the control, miR-494 mimic, and anti-miR-494 groups, respectively. Compared with the control group, miR-494 mimic transfection significantly inhibited HeLa cells invasiveness $(\mathrm{P}<0.01)$, and transfection with anti-miR-494 
significantly increased the ability of HeLa cell invasion $(\mathrm{P}<0.05)$.

miR-494 target gene prediction. TargetScan prediction software indicated that SOCS6 may be a target gene of miR-494. The forecasting SOCS6 3'-UTR target sites are presented in Fig. 5A. The luciferase reporter gene assay demonstrated that luciferase activity was significantly decreased when co-transfected with wild-type pMIR-SOCS6 3'-UTR carrier and miR-494 ( $\mathrm{P}<0.01$; Fig. 5B). Following co-transfection of mutant pMIR-SOCS6 3'-UTR carrier and miR-494, there was no significant difference was identified, suggesting an interaction between miR-494 and SOCS6.

miR-494 mimic transfection increases the expression of SOCS6. As presented in Fig. 6, when compared with NC, miR-494 mimic transfection significantly increased SOCS6 expression in HeLa cells $(\mathrm{P}<0.01)$, while anti-miR-494 transfection significantly inhibited SOCS6 $(\mathrm{P}<0.01)$.

\section{Discussion}

miRNAs are endogenous non-coding RNAs that contain between 19 and 21 nucleotides. miRNAs are involved in regulating a number of biological functions including cell cycle, proliferation, differentiation and apoptosis. Previous studies have demonstrated that a variety of miRNAs exhibit abnormal expression levels in tumor tissue. Yanaihara et al (13) explored miRNA expression in $>100$ cases of primary malignant tumor and adjacent normal tissues. A total of 43 miRNAs exhibited altered expression levels in primary malignant tumor and cancerous tissues, whereby 28 miRNAs were downregulated, and 15 were upregulated (13). Cervical cancer is one of the most common types of gynecologic cancer, and is the fourth leading cause of cancer-associated mortality among females worldwide (14). Previous studies have demonstrated that miRNAs may be abnormally expressed in cervical cancer and this is associated with the prognosis of cervical cancer $(15,16)$. Zhao et al (17) validated that the expression level of miR-491-5p was significantly decreased in cervical cancer and that miR-491-5p inhibited cancer cell proliferation, primarily by regulating human telomerase reverse transcriptase. Furthermore, Deng et al (18) revealed that the expression level of miR-142-3p was significantly decreased in cervical cancer, and that miR142-3p inhibited cancer cell proliferation and invasion, primarily by regulating Frizzled-7.

Previous studies have identified that miR-494 is associated with the development of tumors $(8,9)$. However, there are a limited number of studies on the expression and the function of miR-494 in cervical cancer. The present study was performed on 40 cases of cervical cancer, 40 cases of cervical intraepithelial lesions and 40 cases of normal cervical tissue. The results of the present study determined the expression of miR-494, which demonstrated that miR-494 was significantly decreased in cervical cancer, compared with the other two types of tissue $(\mathrm{P}<0.01)$. In addition, the expression level of miR-494 in cervical cancer tissue was associated with clinical stage, depth of stromal invasion and lymph node metastasis $(\mathrm{P}<0.01)$; however, no associated was determined between miR-494 expression level and the patient's age, tumor diameter and menopause $(\mathrm{P}>0.05)$. The results of the present study suggested that decreased expression of miR-494 is associated with the development of cervical cancer. Furthermore, transfection was performed in the present study which demonstrated that miR-494 mimic transfection significantly inhibited HeLa cell viability and invasion $(\mathrm{P}<0.01)$. Transfection with anti-miR-494 significantly increased the HeLa cell viability and invasion $(\mathrm{P}<0.05)$. These results suggest that, miR-494 may serve a function in the development of cervical cancer by regulating the viability and invasiveness of cancer cells.

miRNAs exhibit biological effects primarily by adjusting the downstream target genes; therefore, the role of downstream target genes determine the function of miRNAs. A previous study has demonstrated that miR-494 regulates the PTEN/protein kinase B signaling pathway, which results in the regulation of glioma cell proliferation, invasion and migration (19). Sun et al (20) identified that miR-494 may effect colorectal cancer cell migration and invasion by directly targeting PTEN. Additionally, in breast cancer, miR-494 exerts effects on the Wnt/ $\beta$-catenin signaling pathway, which inhibits breast cancer (21). Accordingly, in the present study, target genes of miR-494 were selected using TargetScan software. Previous studies $(22,23)$ have identified SOCS6 to exhibit functions in the progression of human cancers, which prompted the focus on this gene.

A previous study demonstrated that $\mathrm{miR}-17-5 \mathrm{p}$ regulated the proliferation of gastric cancer cells by modulating SOCS6 expression (24). Therefore, the present study investigated the expression of SOCS6 in HeLa cells, which demonstrated that miR-494 mimic transfection increased SOCS6 expression $(\mathrm{P}<0.05)$ and anti-miR-494 transfection significantly inhibited SOCS6 expression $(\mathrm{P}<0.05)$. The results of the present study identified SOCS6 as a downstream target gene of miR-494.

The results of the present study demonstrated that the expression of miR-494 was significantly decreased in cervical cancer, and miR-494 inhibited cancer cell proliferation and invasion by regulating the expression of SOCS6. The present study may have provided a novel cancer therapeutic target; however, the molecular mechanism underlying miR-494-induced inhibition of cancer cell proliferation and invasion remains unknown.

\section{References}

1. Torre LA, Bray F, Siegel RL, Ferlay J, Lortet-Tieulent J and Jemal A: Global cancer statistics, 2012. CA Cancer J Clin 65: 87-108, 2015

2. Zhao S, Yao D, Chen J, Ding N and Ren F: MiR-20a promotes cervical cancer proliferation and metastasis in vitro and in vivo. PLoS One 10: e0120905, 2015.

3. Handler AS, Henderson VA, Rosenfeld A, Rankin K, Jones B and Issel LM: Illinois breast and cervical cancer program: Implementing effective public-private partnerships to assure population health. J Public Health Manag Pract 21: 459-466, 2015.

4. Wen Z, Lei Z, Jin-An M, Xue-Zhen L, Xing-Nan Z and Xiu-Wen D: The inhibitory role of miR-214 in cervical cancer cells through directly targeting mitochondrial transcription factor A (TFAM). Eur J Gynaecol Oncol 35: 676-682, 2014.

5. Xu J, Zhang W, Lv Q and Zhu D: Overexpression of miR-21 promotes the proliferation and migration of cervical cancer cells via the inhibition of PTEN. Oncol Rep 33: 3108-3116, 2015.

6. Chen Y, Ma C, Zhang W, Chen Z and Ma L: Down regulation of miR-143 is related with tumor size, lymph node metastasis and HPV16 infection in cervical squamous cancer. Diagn Pathol 9: 88, 2014. 
7. Wang Q, Qin J, Chen A, Zhou J, Liu J, Cheng J, Qiu J and Zhang J: Downregulation of microRNA-145 is associated with aggressive progression and poor prognosis in human cervical cancer. Tumour Biol 36: 3703-3708, 2015.

8. Yang YK, Xi WY,XiRX,Li JY,Li Q and Gao YE: MicroRNA-494 promotes cervical cancer proliferation through the regulation of PTEN. Oncol Rep 33: 2393-2401, 2015.

9. Yuan J, Wang K and Xi M: MiR-494 inhibits epithelial ovarian cancer growth by targeting c-Myc. Med Sci Monit 22: 617-624, 2016.

10. FIGO staging for carcinoma of the vulva, cervix and corpus uteri: International journal of gynaecology and obstetrics: The official organ of the International Federation of Gynaecology and Obstetrics 125: 97-98, 2014.

11. Zhou RP, Chen G, Shen ZL and Pan LQ: Cinobufacin suppresses cell proliferation via miR-494 in BGC-823 gastric cancer cells. Asian Pac J Cancer Prev 15: 1241-1245, 2014.

12. Zou, Ruanmin Hu, Zhi Chen, Hao et al: MiR199a in cervical cancer and cervical intraepithelial lesions Expression and significance. J Med Res 40: 55-59, 2011.

13. Yanaihara N, Caplen N, Bowman E, Seike M, Kumamoto K, Yi M, Stephens RM, Okamoto A, Yokota J, Tanaka T, et al: Unique microRNA molecular profiles in lung cancer diagnosis and prognosis. Cancer Cell 9: 189-198, 2006.

14. Jemal A, Bray F, Center MM, Ferlay J, Ward E and Forman D: Global cancer statistics. CA Cancer J Clin 61: 69-90, 2011.

15. Sharma G, Dua P and Agarwal SM: A comprehensive review of dysregulated miRNAs involved in cervical cancer. Curr Genomics 15: 310-323, 2014.

16. Villegas-Ruiz V, Juárez-Méndez S, Pérez-González OA, Arreola H, Paniagua-García L, Parra-Melquiadez M, Peralta-Rodríguez R, López-Romero R, Monroy-García A, Mantilla-Morales A, et al: Heterogeneity of microRNAs expression in cervical cancer cells: Over-expression of miR-196a. Int J Clin Exp Pathol 7: 1389-1401, 2014.

17. Zhao Q,Zhai YX, Liu HQ, Shi YA and Li XB: MicroRNA-491-5p suppresses cervical cancer cell growth by targeting hTERT. Oncol Rep 34: 979-986, 2015.
18. Deng B, Zhang Y, Zhang S, Wen F, Miao Y and Guo K: MicroRNA-142-3p inhibits cell proliferation and invasion of cervical cancer cells by targeting FZD7. Tumour Biol 36: 8065-8073, 2015

19. Li XT, Wang HZ, Wu ZW, Yang TQ, Zhao ZH, Chen GL, Xie XS, Li B, Wei YX, Huang YL, et al: miR-494-3p regulates cellular proliferation, invasion, migration, and apoptosis by PTEN/AKT signaling in human glioblastoma cells. Cell Mol Neurobiol 35: 679-687, 2015

20. Sun HB, Chen X, Ji H, Wu T, Lu HW, Zhang Y, Li H and Li YM: miR-494 is an independent prognostic factor and promotes cell migration and invasion in colorectal cancer by directly targeting PTEN. Int J Oncol 45: 2486-2494, 2014.

21. Song L, Liu D, Wang B, He J, Zhang S, Dai Z, Ma X and Wang X: miR-494 suppresses the progression of breast cancer in vitro by targeting CXCR4 through the Wnt/ $\beta$-catenin signaling pathway. Oncol Rep 34: 525-531, 2015.

22. Fang Y, Sun B, Xiang J and Chen Z: MiR-301a promotes colorectal cancer cell growth and invasion by directly targeting SOCS6. Cell Physiol Biochem 35: 227-236, 2015.

23. Tanaka T, Arai M, Jiang X, Sugaya S, Kanda T, Fujii K, Kita K, Sugita K, Imazeki F, Miyashita T, et al: Downregulation of microRNA-431 by human interferon- $\beta$ inhibits viability of medulloblastoma and glioblastoma cells via upregulation of SOCS6. Int J Oncol 44: 1685-1690, 2014.

24. Wu Q, Luo G, Yang Z, Zhu F, An Y, Shi Y and Fan D: miR-17-5p promotes proliferation by targeting SOCS6 in gastric cancer cells. FEBS Lett 588: 2055-2062, 2014. 